\title{
Hydrodynamic dispersion in a self-similar geometry
}

\author{
Sidney Redner $\nmid \ddagger$, Joel Koplik $\dagger$ and David Wilkinson $\dagger$ \\ † Schlumberger-Doll Research, Old Quarry Road, Ridgefield, CT 06877-4108, USA \\ $\ddagger$ Center for Polymer Studies and Department of Physics, Boston University, Boston, MA \\ 02215, USA
}

Received 18 June 1986, in final form 31 October 1986

\begin{abstract}
We investigate the dispersion of a dynamically neutral tracer flowing in a self-similar, hierarchical model of a porous medium. We consider a purely convective limit in which the time for tracer particles to traverse a given bond is strictly proportional to the inverse of the flux in the bond; the effect of molecular diffusion is neglected. Our hierarchical model contains an adjustable 'asymmetry' parameter which controls the width of the distribution of transit times for a tracer to traverse the multiplicity of paths in the network. We derive a functional recursion relation for this distribution, from which exact expressions for the moments can be obtained. We find two regimes of behaviour which are governed by the value of the asymmetry parameter. In one regime, the transit time distribution is characterised by a single timescale, so that a localised pulse of tracer spreads out at the same rate at which the pulse is convected downstream. In the second regime, the small fraction of tracer passing through the slowest bond of the network dominates in the moments of the distribution, leading to highly enhanced dispersion. The consequences of these results for the dispersion coefficient are discussed, both for self-similar and homogeneous systems.
\end{abstract}

\section{Introduction}

Hydrodynamic dispersion is a fundamental transport process in which a localised pulse of dynamically neutral tracer disperses in a flow field under the combined action of convection and molecular diffusion (for reviews see, e.g., Bear 1971, Fried and Combarnous 1971, Scheidegger 1974). The subtle interplay between these two mechanisms gives rise to a transport phenomenon which is considerably more complicated, but richer phenomenologically, than that of either simple diffusion or biased diffusion. This is particularly true when the background flow is in a randomly porous medium where the typical length scale of the heterogeneities, which plays the role of a correlation length, is very large. In such a situation, the dispersion process can be dominated by the small fraction of tracer that is caught in relatively rare, stagnant regions within the flow field (Saffman 1959, 1960, de Gennes 1983). The understanding of dispersion is therefore of fundamental theoretical interest, as well as being of relevance for applied fields such as oil recovery, hydrology and chromatography. Very recently, there have been a variety of numerical simulation studies of dispersion in porous media models, both in the convective limit (Sahimi et al 1983, 1986, Leitzelment et al 1984), and with the effects of diffusion included (de Arcangelis et al 1986, Michel 1986, Roux et al 1986). These latter studies indicate that stagnation effects are important and that considerable effort is required to treat them properly. 
Given the difficulties inherent in accounting for both convection and molecular diffusion in dispersion phenomena within porous media, it is worthwhile to consider simpler situations which are tractable analytically. In this paper, we consider dispersion in a self-similar hierarchical model in the absence of molecular diffusion-i.e. in the purely convective limit. We introduce an adjustable 'asymmetry' parameter for the model which controls the width of the distribution of transit times for tracer to pass through the multiplicity of fluid paths through the network. (A related model with a fixed asymmetry, a Koch curve, has been introduced by Mandelbrot and Given (1984).) Since the hierarchical model provides an excellent description of the geometry of the percolating backbone near the percolation threshold (de Arcangelis et al 1985), we expect to be able to use our model to describe basic aspects of dispersion on systems with percolation-type disorder in the convective limit.

In $\S 2$, we begin by developing a correspondence between the flow problem in a random network of tubes and the electrical current flow problem in a random resistor network. We next introduce the asymmetric hierarchical model as a description of dispersion phenomena in a self-similar random medium. A number of very simple results for the average rate of transport of tracer particles across the network are given and an exact connection between the average transit time and the critical properties of the conductivity and the backbone density is made.

In $\S 3$, we calculate the distribution of transit times that arises when an ensemble of tracer particles is convected through the network. Due to the relative simplicity of the hierarchical model, we can derive an exact formal expression for the moments of the transit time distribution. As the asymmetry of the hierarchical model is varied, the width of the transit time distribution also varies in a corresponding manner, and this leads to two generic classes of behaviour. For a relatively narrow distribution, we find $\left\langle T_{N}^{k}\right\rangle \sim c_{k}\left\langle T_{N}\right\rangle^{k}$, where $\left\langle T_{N}^{k}\right\rangle$ is the $k$ th moment of the transit time distribution on the $N$ th-order hierarchical structure and $c_{k}$ is a numerical coefficient. This implies that a localised pulse of tracer spreads out at the same rate at which the pulse is convected downstream. On the other hand, for a sufficiently broad distribution, we find $\left\langle T_{N}^{2}\right\rangle /\left\langle T_{N}\right\rangle^{2} \rightarrow \infty$ as $N \rightarrow \infty$, leading to an enhanced dispersion process which is dominated by the small fraction of tracer that passes through the slowest bond in the network.

In $\$ 4$, we use the results derived for the moments of the transit time distribution to make predictions for the longitudinal dispersion coefficient, $D_{\|}$. We first consider $D_{\|}$for a homogeneous system, obtained by superposing many hierarchical structures in a linear sequence. Thus the system length can be made much larger than the correlation length (the length of one hierarchical structure), and this should provide a useful picture for dispersion in a percolating system above the percolation threshold. For such a system, the hypotheses of the central limit theorem will apply, so that the tracer distribution ultimately becomes a Gaussian. When the transit time distribution on one hierarchical structure is sufficiently narrow, $D_{\|}$is found to be proportional to $U L$, where $U$ is the average flow velocity, and $L$ is the effective length of the hierarchical model. Thus $D_{\|}$has the same qualitative form as in porous media with microscopic disorder, where it is well known that $D_{\|} \sim U l$, with $l$ being a characteristic pore or grain size in the medium. In the case of a broad transit time distribution, we show that $D_{\|}$is proportional to $U L^{\alpha}$, where the exponent $\alpha$ may take on any value greater than unity. In addition, we address the question of how long the system must be before Gaussian behaviour will set in

Next we consider dispersion in the self-similar regime, i.e. at the scale of a single hierarchical structure. This should provide a valid picture of dispersion on a random 
medium which is at the percolation threshold. In this regime, $D_{\|}$is scale dependent, and this leads to anomalous dispersion, in close analogy with anomalous diffusion on self-similar systems (Gefen et al 1982). The consequences of this phenomenon for the critical behaviour of the dispersion coefficient are discussed.

A brief summary and discussion is given in $\S 5$.

\section{Transport properties of the asymmetric hierarchical model}

To model dispersion in random media, we appeal to the analogy between Poiseuille flow in a random tube network and current flow in a random resistor network. In terms of the random resistor network, when an external potential is applied across the system, the steady-state currents in the bonds define the background flow field for the corresponding fluid flow problem.

In the flow problem, it proves to be most convenient to adopt a parametrisation which gives a bond transit time varying exactly as the inverse of the flux in the bond, and in which all the bonds in the network have the same volume. As we shall see, this ensures that tracer spends an equal amount of time, on average, in each bond of the network. For a resistor with conductance $\varepsilon$, we choose the radius $r$ and the length $l$ of the corresponding tube by $r^{2} \sim \varepsilon^{1 / 3}$ and $l \sim \varepsilon^{-1 / 3}$. This gives a flow conductance, $g \sim r^{4} / l$, varying as $\varepsilon$. In addition, when a unit pressure drop is imposed across the bond, the average fluid velocity $v \sim g / r^{2}$ varies as $\varepsilon^{2 / 3}$, and the transit time $\tau \sim l / v$ therefore varies as $1 / \varepsilon$. Thus we have constructed a model in which the bond transit time is strictly proportional to the inverse of the flux in the bond.

We now exploit this construction to describe dispersion on an asymmetric hierarchical model where an adjustable parameter $\varepsilon$ controls the width of the distribution of transit times for tracer particles to convect across the network (figure 1). Topologically, the model is a hierarchical embedding of both singly corrected bonds and blobs; this appears to capture many of the essential geometrical features of the percolating backbone at the percolation threshold (de Arcangelis et al 1985). A new aspect of the asymmetric version of the model is that the curved bonds at a given level have a conductance decreased by a factor $\varepsilon$ (and hence a transit time increased by a factor $1 / \varepsilon$ ), compared to the straight bonds at the same level.

In the convective limit, the tracer can enter a particular bond with a probability proportional to the flux in the bond, and then the tracer moves with the cross-sectional average velocity until reaching the next node (Sahimi et al 1983, 1986, de Arcangelis et al 1986). When $\varepsilon=1$ (symmetric limit), both paths of the first-order structure have the same transit time and there is no dispersion, while as $\varepsilon \rightarrow 0$, one fluid path will have a diverging transit time while the other transit time remains finite. For a general $N$ th-order structure with $\varepsilon \neq 1$, the variety of distinct paths available for the tracer causes a delta function input of tracer at one end of the hierarchical medium to evolve into a broadened distribution at the outlet end.

Before discussing dispersion, let us first outline some basic geometrical and transport properties of the hierarchical model. For an $N$ th-order symmetric structure, it is straightforward to verify that the conductance, $G$, equals $\left(\frac{2}{3}\right)^{N}$, that the number of bonds in the backbone, $N_{\mathrm{BB}}$, equals $4^{N}$ and that the number of singly connected bonds, $N_{\mathrm{sc}}$, equals $2^{N}$. In order to make contact with critical exponents, it is necessary to infer an effective linear dimension for the $N$ th-order model. One natural assignment is based on the fundamental exact result for percolation clusters at the threshold, 
(a)
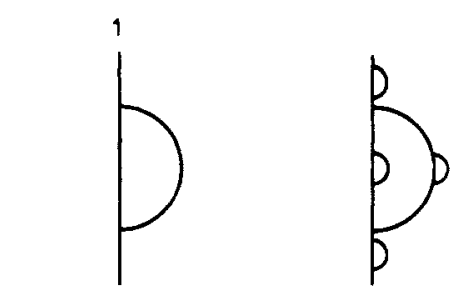

(b)
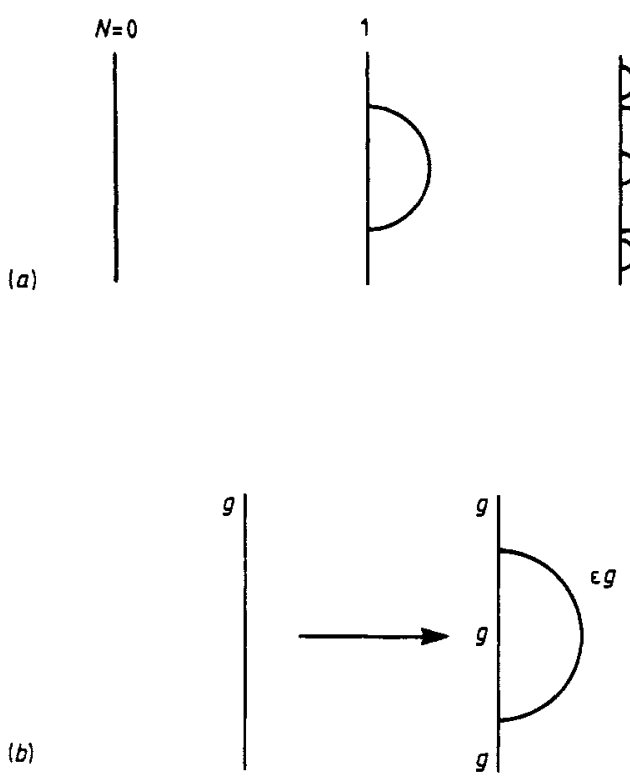

Figure 1. (a) The self-similar hierarchical model. Shown is the lattice at $N=0,1$ and 2 levels of iteration. $(b)$ In the hierarchical construction, a bond of conductance $g$ at level $N$ is replaced at level $N+1$ by four new bonds with conductances as shown.

$N_{\mathrm{sc}} \sim L^{1 / \nu}$, with $L$ being the linear dimension of the system and $\nu$ being the correlation length exponent (Coniglio 1981, 1982). Since $N_{\mathrm{sc}}=2^{N}$ in the $N$ th-order model, we therefore identify an effective linear dimension through $L=2^{N \nu}$. Eliminating $N$ in favour of $L$ we thereby find that $G$ varies as $L^{-t / \nu}$ with a conductivity exponent $t$ equal to $\ln \frac{5}{2} / \ln 2 \simeq 1.3219 \ldots$, while the backbone fraction, i.e. $N_{\mathrm{BB}}$ divided by the volume $L^{d}$, varies as $L^{-\beta_{\mathrm{BB}} / \nu}$, with $\beta_{\mathrm{BB}}=d \nu-\ln 4 / \ln 2=2(\nu-1)$. In the last relation, $d$ is the spatial dimension which we have taken to be equal to 2 . These exponents are consistent with known results, assuming the generally accepted value of $\nu=\frac{4}{3}$ in two dimensions. This provides a useful check on the validity of our model as a description of the geometry of the backbone near the percolation threshold. For the asymmetric model, however, the relation $L=2^{N \nu}$, and the exponent results which follow, may no longer have the same degree of plausibility as in the symmetric model.

Let us now consider the average time required for tracer to traverse the $N$ th-order structure, $\left\langle T_{N}\right\rangle$, when a unit potential difference is imposed across the two endpoints of the model. By decomposing the $N$ th-order structure into the four $(N-1)$ th-order structures as indicated in figure 2, the transit times on each of the lower-order structures, relative to the transit time when a unit external potential drop is applied across each structure, will be rescaled by the factors $a \equiv(3+2 \varepsilon) /(1+\varepsilon), b \equiv 3+2 \varepsilon, a$ and $c \equiv$ $(3+2 \varepsilon) / \varepsilon$, respectively. Since the tracer takes the straight path with probability $p_{\mathrm{st}}=1 /(1+\varepsilon)$, and the curved path with probability $p_{\mathrm{cu}}=\varepsilon /(1+\varepsilon)$, we find a simple relation between $\left\langle T_{N}\right\rangle$ and $\left\langle T_{N-1}\right\rangle$ :

$$
\left\langle T_{N}\right\rangle=p_{\mathrm{st}}(2 a+b)\left\langle T_{N-1}\right\rangle+p_{\mathrm{cu}}(2 a+c)\left\langle T_{N-1}\right\rangle \equiv a_{1}\left\langle T_{N-1}\right\rangle
$$

with $a_{1}=4 a$. Using $\left\langle T_{0}\right\rangle=1$, we thus obtain

$$
\left\langle T_{N}\right\rangle=\left\langle T_{1}\right\rangle^{N}=\left(a_{1}\right)^{N} \text {. }
$$




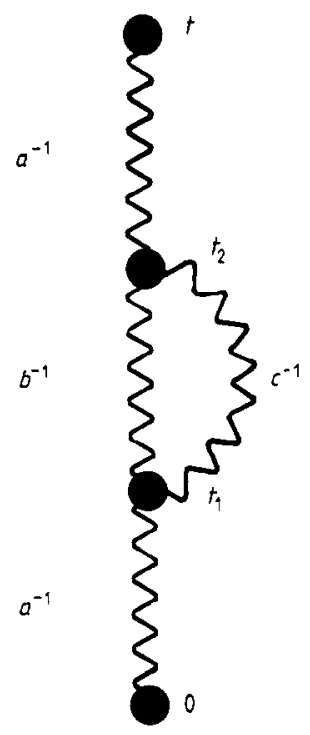

Figure 2. Decomposition of an $N$ th-order structure into four $(N-1)$ th-order structures. The labelling of the times used in the integral recurrence relation $(2 a)$ is indicated. Also shown are the fluxes across each of the lower-order structures.

Our derivation of the average time has fundamental ramifications which hold for any random network of tubes. These features appear to be sufficiently interesting so that a brief digression is worthwhile. For the hierarchical model, notice that from the relation between the entrance probabilities and the transit times, the tracer particle spends an equal amount of time in each bond, on average, independent of the value of $\varepsilon$. More generally, for an arbitrary tube network in which each bond has a distinct volume $\Omega_{i}$, the average amount of time that a tracer particle spends in the bond is proportional to $\Omega_{i}$. To show this, suppose that the total current passing through the network is $I$, and that a current $I_{i}$ is passing through bond $i$, which has length $l_{i}$ and cross-sectional area $a_{i}$. In the convective limit, the probability of tracer entering this bond is simply $I_{i} / l$. Since the flow velocity in bond $i$ is $I_{i} / a_{i}$, the average tracer residence time will be $\left(I_{i} / I\right)\left(l_{i} /\left(I_{i} / a_{i}\right)\right)=\Omega_{i} / I$, that is, the residence time is proportional to the volume of the bond. Then the total average time that the tracer spends in the system, $\langle T\rangle$, is $\Omega / I$, where $\Omega$ is the total volume of the system.

For a percolating network, $\Omega$ can be identified with $N_{\mathrm{BB}}$, while for a unit potential drop, $I$ can be identified with the conductance $G$, leading to $\langle T\rangle$ scaling exactly as $N_{\mathrm{BB}} / G$. This very basic result does not seem to have been mentioned or appreciated previously in the percolation literature. As a useful check of these results, it can be straightforwardly verified that the above relations hold on the asymmetric hierarchical model.

More generally, one might inquire if there is a relation between the higher moments of the transit time distribution and the current distribution in the bonds of the network. While we have been unable to prove a general result, it can be seen on simple networks, on which dispersion occurs, that there is a qualitative relation between the two types of moments. From considering a number of simple examples, we find that the dominant contribution to the $k$ th moment of the transit time is given by the moment of order $1-k$ of the current distribution. 


\section{Transit time distribution on the hierarchical model}

Let us denote by $P_{N}(V ; t)$ the probability density for traversing an $N$ th-order hierarchical structure in a time $t$ when a voltage $V$ is applied across the system. From figure 2 , it is easy to see that $P_{N}(V ; t)$ satisfies the integral recurrence relation

$$
\begin{gathered}
P_{N}(V ; t)=\int_{0}^{t_{2}} \mathrm{~d} t_{1} \int_{0}^{t} \mathrm{~d} t_{2}\left[p_{\mathrm{st}} P_{N-1}\left(\frac{V}{a} ; t_{1}\right) P_{N-1}\left(\frac{V}{b} ; t_{2}-t_{1}\right) P_{N-1}\left(\frac{V}{a} ; t-t_{2}\right)\right. \\
\left.+p_{\mathrm{cu}} P_{N-1}\left(\frac{V}{a} ; t_{1}\right) P_{N-1}\left(\frac{V}{c} ; t_{2}-t_{1}\right) P_{N-1}\left(\frac{V}{a} ; t-t_{2}\right)\right]
\end{gathered}
$$

By introducing the Laplace transform, $\tilde{P}_{N}(V ; z)=\int P_{N}(V ; t) \mathrm{e}^{-z t} \mathrm{~d} t$, and expressing all the $\tilde{P}_{N}$ with respect to a unit voltage, we obtain the functional recursion relation

$$
\tilde{P}_{N}(z)=\left[\tilde{P}_{N-1}(a z)\right]^{2}\left(p_{\mathrm{st}} \tilde{P}_{N-1}(b z)+p_{\mathrm{cu}} \tilde{P}_{N-1}(c z)\right)
$$

where the argument $V(=1)$ has been dropped since it is identical in all factors of $P$.

On the other hand, we can write the Laplace transform as a moment generating function

$$
\tilde{P}_{N}(z)=\left\langle\mathrm{e}^{-z t}\right\rangle=1-z\left\langle T_{N}\right\rangle+\frac{1}{2} z^{2}\left\langle T_{N}^{2}\right\rangle-\ldots
$$

so that by expanding $(2 b)$ in a power series, we find the following recursion relation for the moments

$$
\frac{\left\langle T_{N}^{k}\right\rangle}{k !}=\sum_{\substack{\alpha, \beta, \gamma \\ \alpha+\beta+\gamma=k}}^{k}\left\langle T_{N-1}^{\alpha}\right\rangle\left\langle T_{N-1}^{\beta}\right\rangle\left\langle T_{N-1}^{\gamma}\right\rangle \frac{a^{\alpha+\beta}}{\alpha ! \beta ! \gamma !}\left[p_{\mathrm{st}} b^{\gamma}+p_{\mathrm{cu}} c^{\gamma}\right] .
$$

Thus the $k$ th moment on the $N$ th-order structure can be decomposed in terms of all moments up to order $k$ on the $(N-1)$ th-order structure. To appreciate the consequences of this decomposition, consider the special case $k=2$. From (4) we obtain

with

$$
\left\langle T_{N}^{2}\right\rangle=a_{2}\left\langle T_{N-1}^{2}\right\rangle+b_{2}\left\langle T_{N-1}\right\rangle^{2}
$$

$$
\begin{aligned}
& a_{2}=\left(\frac{1+4 \varepsilon+\varepsilon^{2}}{\varepsilon}\right)\left(\frac{3+2 \varepsilon}{1+\varepsilon}\right)^{2} \\
& b_{2}=10\left(\frac{3+2 \varepsilon}{1+\varepsilon}\right)^{2}
\end{aligned}
$$

If we iterate (5), we ultimately arrive at

$$
\left\langle T_{N}^{2}\right\rangle=\left(1-c_{2}\right) a_{2}^{N}+c_{2}\left(a_{1}^{2}\right)^{N}
$$

with

$$
c_{2}=\frac{b_{2}}{a_{1}^{2}-a_{2}}=\frac{10 \varepsilon}{12 \varepsilon-\varepsilon^{2}-1} .
$$

When $a_{1}^{2}>a_{2}$, which occurs when $\varepsilon>0.08392 \ldots \equiv \varepsilon_{2}$, the second term in (7) dominates and we have, as $N \rightarrow \infty$

$$
\left\langle T_{N}^{2}\right\rangle \sim c_{2}\left(a_{1}^{2}\right)^{N}=c_{2}\left\langle T_{N}\right\rangle^{2}
$$

Notice, in particular, that $\left\langle T_{N}^{2}\right\rangle=\left\langle T_{N}\right\rangle^{2}$ when $\varepsilon=1$, since there is no dispersion in this case. Thus we have found that when $\varepsilon>\varepsilon_{2}$, both $\left\langle T_{N}\right\rangle$ and $\left\langle T_{N}^{2}\right\rangle$ are governed by a single characteristic time as $N \rightarrow \infty$. 
However for $\varepsilon \leqslant \varepsilon_{2}$, the first term in (7) dominates, so that as $N \rightarrow \infty$

$$
\left\langle T_{N}^{2}\right\rangle \sim\left(1-c_{2}\right) a_{2}^{N}
$$

i.e. $\left\langle T_{N}^{2}\right\rangle \gg\left\langle T_{N}\right\rangle^{2}$. The limiting behaviour for the second (and higher) moments as $N \rightarrow \infty$ and $\varepsilon \rightarrow 0$ can be interpreted simply in terms of the small fraction of tracer which enters the slowest bond of the network. That is, taking the time required to traverse the slowest bond in the network and multiplying by the probability of entering this bond gives a contribution to the second moment equal to $(9 / \varepsilon)^{N}$. This coincides with (9) as $\varepsilon \rightarrow 0$, demonstrating that the second moment is indeed dominated by the tracer which passes through the slowest bond in the network. Moreover, as $\varepsilon \rightarrow 0$, $\left\langle T_{N}\right\rangle \sim 12^{N}$, so that the ratio $\left\langle T_{N}^{2}\right\rangle /\left\langle T_{N}\right\rangle^{2}$ diverges as $(16 \varepsilon)^{-N}$. This demonstrates that there is more than one typical timescale that characterises the transit time distribution.

Very similar, but progressively more tedious, considerations can be applied to the higher moments. As in the case $k=2$, we can decompose the $k$ th moment in terms of lower-order moments, by following steps similar to those given in (4)-(7), to give an expression of the following form

$$
\begin{array}{r}
\left\langle T_{N}^{k}\right\rangle=c_{k}\left(a_{1}^{k}\right)^{N}+d_{k}\left(a_{1}^{k-2} a_{2}\right)^{N}+\ldots+w_{k}\left(a_{2} a_{k-2}\right)^{N} \\
+x_{k}\left(a_{1}^{2} a_{k-2}\right)^{N}+y_{k}\left(a_{1} a_{k-1}\right)^{N}+z_{k} a_{k}^{N}
\end{array}
$$

with

$$
a_{k}=a^{k}\left[2+(1+\varepsilon)^{k-1}+\left(\frac{1+\varepsilon}{\varepsilon}\right)^{k-1}\right]
$$

where $c_{k}, d_{k}, \ldots$ are numerical coefficients, and in each term in (10), the sum of the values of the subscripts of all factors of $a_{i}$ must equal $k$. The asymptotic $N \rightarrow \infty$ behaviour $\left\langle T_{N}^{k}\right\rangle$ will be given by the largest term in (10), and we can determine which of these terms dominates by comparing $a_{k}$ with $a_{1}^{k}$. Thus define $\varepsilon_{k}$ to be the root of the equation $a_{k}=a_{1}^{k}$, i.e. the zero of

$$
4^{k}-2-(1+\varepsilon)^{k-1}-\left(\frac{1+\varepsilon}{\varepsilon}\right)^{k-1}
$$

The $\varepsilon_{k}$ form an increasing sequence with $\varepsilon_{2}=0.08392 \ldots, \varepsilon_{3}=0.14727 \ldots, \varepsilon_{4}=$ $0.188003 \ldots$, etc, with $\lim _{k \rightarrow \infty} \varepsilon_{k}=\frac{1}{3}$. In terms of the $\varepsilon_{k}$, we find that either the first term in (10) dominates, for $\varepsilon>\varepsilon_{k}$, or that the last term dominates for $\varepsilon \leqslant \varepsilon_{k}$. While we have not been able to construct a general proof of this fact, it has been verified explicitly for $k=2$ and $k=3$ and verified numerically for larger values of $k$.

Thus the general picture that emerges from our analysis is that for $\varepsilon>\frac{1}{3}$, the relation

$$
\left\langle T_{N}^{k}\right\rangle \sim c_{k}\left\langle T_{N}\right\rangle^{k}
$$

holds for all $k$. That is, there is a single timescale, $\left\langle T_{N}\right\rangle$, which characterises all the moments of the transit time distribution. We term this regime of behaviour the weakly asymmetric limit. For $\varepsilon_{k}<\varepsilon \leqslant \varepsilon_{k+1}$, moments of order $j<k$, continue to behave as in (13), namely

$$
\left\langle T^{j}\right\rangle \sim c_{j}\left(a_{1}^{j}\right)^{N}=c_{j}\left\langle T_{N}\right\rangle^{j}
$$

while moments of order $j \geqslant k$, behave as

$$
\left\langle T_{N}^{j}\right\rangle \sim z_{j} a_{j}^{N}
$$


i.e. $\left\langle T_{N}^{j}\right\rangle \gg\left\langle T_{N}\right\rangle^{j}$. Thus only at a sufficiently high order does each moment scale independently. As in the case of the second moment, this independent scaling behaviour arises solely from the small portion of the tracer which enters the slowest bond in the network. When $\varepsilon \rightarrow 0$, this tracer fraction gives a contribution to the $k$ th moment which varies as

$$
\left(\frac{3^{k}}{\varepsilon^{(k-1)}}\right)^{N}
$$

and this coincides with the behaviour of $a_{k}^{N}$ in the limit $\varepsilon \rightarrow 0$. Finally for $\varepsilon<\varepsilon_{2}$, all the moments scale independently, as moments beyond the first are dominated by the contribution of the tracer which traverses the slowest bond in the network. We term this regime of behaviour the strongly asymmetric limit.

In the weakly asymmetric limit, the single timescale relation (13) permits us to write a general recursion relation for the coefficients $c_{k}$. Using (13) in (4), and defining $D_{k}=c_{k} / k$ !, we find

$$
D_{k}=\sum_{\substack{\alpha, \beta, \gamma \\ \alpha+\beta+\gamma=k}}^{k} D_{\alpha} D_{\beta} D_{\gamma} a^{\alpha+\beta}\left[p_{\mathrm{st}} b^{\gamma}+p_{\mathrm{cu}} c^{\gamma}\right]
$$

By moving those terms where one of $\alpha, \beta$ or $\gamma$ equals $k$ to the left-hand side, we obtain

$$
D_{k}=\sum_{\alpha, \beta, \gamma<k} D_{\alpha} D_{\beta} D_{\gamma}\left[(1+\varepsilon)^{\gamma-1}+\left(\frac{1+\varepsilon}{\varepsilon}\right)^{\gamma-1}\right]\left[4^{k}-2-(1+\varepsilon)^{k-1}-\left(\frac{1+\varepsilon}{\varepsilon}\right)^{k-1}\right]^{-1} \text {. }
$$

We see that the condition for the denominator to remain positive for all $k$ coincides with the condition $\left.a_{1}^{k}\right\rangle a_{k}$ which is required to give $\left\langle T_{N}^{k}\right\rangle$ scaling as $\left\langle T_{N}\right\rangle^{k}$.

The single timescale relation (13) may also be used to simplify the form of the Laplace transform of the transit time distribution. Thus using (13) in (3) yields, for large $N$

$$
\begin{aligned}
\tilde{P}_{N}(z)=\left\langle\mathrm{e}^{-z t}\right\rangle & =1-z\left\langle T_{N}\right\rangle+\frac{1}{2} c_{2} z^{2}\left\langle T_{N}\right\rangle^{2}-\ldots \\
& \equiv \tilde{g}\left(z\left\langle T_{N}\right\rangle\right) .
\end{aligned}
$$

That is, $\tilde{P}_{N}(z)$ is a function only of the combination $z\left\langle T_{N}\right\rangle=z(4 a)^{N}$. Using (18) in $(2 b)$, we find the following functional relation for the scaling function $\tilde{g}(z)$

$$
\tilde{g}(z)=\left[\tilde{g}\left(\frac{z}{4}\right)\right]^{2}\left[p_{\mathrm{st}} \tilde{g}\left(\frac{b z}{4 a}\right)+p_{\mathrm{cu}} \tilde{g}\left(\frac{c z}{4 a}\right)\right]
$$

with asymptotic solution as $z \rightarrow \infty$

$$
\tilde{g}(z) \sim A \exp \left(-\left(z / z_{0}\right)^{\alpha}\right)
$$

where $A=(1+\varepsilon)^{1 / 2}, z_{0}$ is arbitrary $\dagger$, and $\alpha$ is determined by the root of $4^{\alpha}=2+(1+\varepsilon)^{\alpha}$. When $\varepsilon=1$, this gives $\alpha=1$, which corresponds to a delta function for the transit time distribution. On the other hand, for $\varepsilon<1, \alpha$ is also less than 1 , and this quasiexponential decay in $z$ implies a broadened distribution of transit times.

From the large- $z$ behaviour in the Laplace domain, we can obtain information about the small-time behaviour of the transit time distribution. Using the scaling form 
(18) in the inverse Laplace transform, we find that $P_{N}(t)$ behaves as

$$
P_{N}(t) \sim \frac{1}{\left\langle T_{N}\right\rangle} g\left(\frac{t}{\left\langle T_{N}\right\rangle}\right)
$$

where $g$ is the inverse Laplace transform of $\tilde{g}$. By steepest descents, we find that for $t \ll 1, g(t)$ varies as

$$
g(t) \sim t^{x} \mathrm{e}^{-(t / \tau)^{-y}}
$$

with

$$
y=\frac{\alpha}{1-\alpha} \quad x=-\left(\frac{1-\alpha / 2}{1-\alpha}\right)
$$

and

$$
\tau=\left[\left(\frac{\alpha}{1-\alpha}\right)^{1-\alpha}-\left(\frac{\alpha}{1-\alpha}\right)^{-\alpha}\right]^{-1 / \alpha}
$$

The large-time behaviour of the transit time distribution is more difficult to determine. However, it is possible to establish that the distribution decays faster than any power law as $t \rightarrow \infty$. A quasi-exponential decay of the form $g(t) \sim B t^{\gamma} \exp \left(-\left(t / t_{0}\right)^{\beta}\right)$ was found to give moments which very roughly approximated those of the hierarchical model for appropriate values of $B, \beta$, and $\gamma$. Because this fitting procedure was not entirely successful, it suggests that the functional form of the transit time distribution is more complicated than a single quasi-exponential decay.

\section{The dispersion coefficient}

Let us now relate our results for the moments of the transit time distribution to the dispersion coefficient. For a one-dimensional homogeneous system, the macroscopic description for the spreading of tracer in a flow field is provided by the convectivediffusion equation (CDE),

$$
\frac{\partial c}{\partial t}+U \frac{\partial c}{\partial x}=D_{\|} \frac{\partial^{2} c}{\partial x^{2}}
$$

where $c$ is the tracer concentration, $U$ is the average flow velocity, defined to be in the $x$ direction and $D_{\|}$is the (macroscopic) longitudinal dispersion coefficient. In the convective limit, $D_{\|}$is related to the transit time moments by

$$
D_{\|}=\left(U^{3} / 2 L\right) \sigma_{t}^{2}
$$

where $L$ is the system length, $\sigma_{t}^{2}=\left\langle t^{2}\right\rangle-\langle t\rangle^{2}$ and $\left\langle t^{k}\right\rangle$ is the $k$ th moment of the transit time across the system (see, e.g., Sahimi et al 1983, 1986). According to this macroscopic description, a point source of tracer will ultimately evolve into a Gaussian wavepacket as it is convected downstream. This Gaussian behaviour is predicated on the homogeneity of the system, so that for a sufficiently long sample it will be possible to invoke the central limit theorem.

A single hierarchical model is not described by a convection-diffusion equation, however, since the model is self-similar on all scales, and the correlation length $\xi$ coincides with the system length $L$. To achieve a homogeneous system, therefore, we consider a linear sequence of hierarchical structures. We can view this linear chain as one of the sequences of macrolinks that comprise the nodes and links picture of 
the percolating cluster. Now the system length can be made much larger than the correlation length, so that Gaussian behaviour will ultimately set in with a finite dispersion coefficient, whose value is correlation length dependent. This series construction should be appropriate for accounting for dispersion in a percolating medium above the percolation threshold.

Thus consider $M$ hierarchical lattices of order $N$ in series (figure 3 ). The transit time distribution $\rho(t)$ across the composite structure is a convolution of $M$ distributions $P_{N}\left(t_{i}-t_{i-1}\right)$ from a single $N$ th-order hierarchy, and the Laplace transform satisfies

$$
\rho(z)=\left[P_{N}(z)\right]^{M} \text {. }
$$

The first two moments of the transit time distribution are then

$$
\begin{aligned}
& \langle t\rangle=-\rho^{\prime}(0)=M\left\langle T_{N}\right\rangle \\
& \left\langle t^{2}\right\rangle=\rho^{\prime \prime}(0)=M\left[\left\langle T_{N}^{2}\right\rangle-\langle T\rangle^{2}\right]+M^{2}\left\langle T_{N}\right\rangle^{2}
\end{aligned}
$$

so that $\sigma_{t}^{2}=M \sigma_{T_{N}}^{2}$, where $\sigma_{T_{N}}^{2}$ is the dispersion on one hierarchical model. Note from (24) that the dispersion coefficient of the linear structure shown in figure 3 is simply given by

$$
D_{\|}=\frac{U}{2(M L)}\left(M \sigma_{T_{N}}^{2}\right)=\frac{U^{3}}{2 L} \sigma_{T_{N}}^{2} .
$$

Thus, provided that the CDE (23) applies to the series structure, the dispersion coefficient can be correctly computed from a single hierarchy alone.

From (27), there are two possible forms for the dispersion coefficient, which depend on the degree of asymmetry of the hierarchica! model. For the weakly asymmetric model, we have $\left\langle T_{N}^{k}\right\rangle \sim c_{k}\left\langle T_{N}\right\rangle^{k}$ with $c_{k} \neq 1$, so that $\sigma_{T_{N}}^{2} \sim\left\langle T_{N}\right\rangle^{2}$. Using $U\left\langle T_{N}\right\rangle=L$, we obtain from (24)

$$
D_{\|} \sim U L \text {. }
$$

This should be compared to the relation $D_{\| j} \sim U l$ which describes dispersion in the convective limit for a homogeneous porous medium with an average grain size equal to $l$ (see, e.g., Bear 1971). Thus to find the dispersion coefficient in a random medium in which the scale of the heterogeneities is $L$, the replacement $l \rightarrow L$ is indicated. This is analogous to the result given by de Gennes (1983) for dispersion on the percolating backbone with the effects of molecular diffusion accounted for, where a naive replacement of a microscopic length with the correlation length suffices to give the desired result.

On the other hand, for the strongly asymmetric hierarchical model, we have $\left\langle T_{N}^{2}\right\rangle \sim\left\langle T_{N}\right\rangle^{2} /(16 \varepsilon)^{N} \gg\left\langle T_{N}\right\rangle^{2}$ as $\varepsilon \rightarrow 0$. Using this relation, we can now write the dispersion coefficient as

$$
D_{\|} \sim U L \frac{\left\langle T_{N}^{2}\right\rangle}{\left\langle T_{N}\right\rangle^{2}}
$$

We can compare this expression to (28) more clearly by using $L=2^{N_{\nu}}$ to write

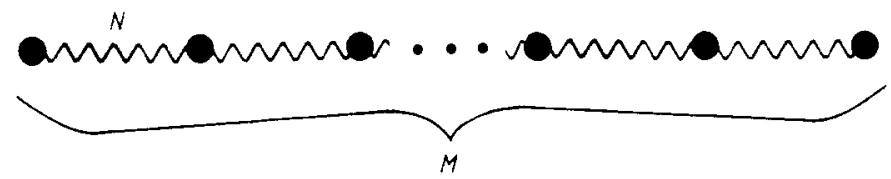

Figure 3. A linear sequence of hierarchical models which yields a system that is described by a one-dimensional convective-diffusion equation. 
$\left\langle T_{N}^{2}\right\rangle /\left\langle T_{N}\right\rangle^{2}$ as $L^{y / \nu}$ with $y=-\ln 16 \varepsilon / \ln 2 \gg 0$. This then yields a non-linear dependence of $D_{\|}$on $L$ :

$$
D_{\|} \sim U L^{(1+y / \nu)} .
$$

Therefore useful information about the nature of the microscopic transit time distribution can be obtained from the $L$ dependence of the macroscopic dispersion coefficient.

As a test of the validity of the macroscopic description for dispersion on a linear sequence of hierarchical models, we now examine the skewness of the transit time distribution:

$$
S(M, \varepsilon)=\frac{\left\langle t^{3}\right\rangle-3\langle t\rangle \sigma_{t}^{2}-\langle t\rangle^{3}}{\left(\sigma_{t}^{2}\right)^{3 / 2}}
$$

This quantity is identically zero for a Gaussian distribution, and it should therefore vanish when the series structure is long enough. In our series construction, we find

$$
\left\langle t^{3}\right\rangle=M\left\langle T_{N}^{3}\right\rangle+3 M(M-1)\left\langle T_{N}^{2}\right\rangle\left\langle T_{N}\right\rangle+M(M-1)(M-2)\left\langle T_{N}\right\rangle^{3}
$$

so that

$$
S(M, \varepsilon)=M^{-1 / 2} \frac{\left\langle T_{N}^{3}\right\rangle-3\left\langle T_{N}\right\rangle \sigma_{T_{N}}^{2}-\left\langle T_{N}\right\rangle^{3}}{\left(\sigma_{T_{N}}^{2}\right)^{3 / 2}}
$$

This quantity vanishes as $M \rightarrow \infty$, but there is a non-trivial dependence of $S$ on $\varepsilon$. More generally, the deviation of the $k$ th moment from its Gaussian value will be of the form $M^{-(k-2) / 2} f_{k}(\varepsilon)$ and hence these deviations will vanish as $M \rightarrow \infty$. Thus the higher moments eventually coincide with those of a Gaussian distribution, so that a linear sequence of $M$ hierarchical structures can be described by a one-dimensional CDE, when $M$ is sufficiently large.

From (33), we also deduce the number of hierarchical structures needed before Gaussian behaviour is evident. For the weakly asymmetric hierarchical model, the temporal moments in (33) are all of the same order, so that $M \gg 1$ is required for Gaussian behaviour to set in. On the other hand, for the strongly asymmetric model, the skewness varies as $\left(M \varepsilon^{N}\right)^{-1 / 2}$, so that a much more stringent condition, $M \gg \varepsilon^{-N}$, is required for Gaussian behaviour to set in.

Now let us consider dispersion in the self-similar regime for the weakly asymmetric limit. Since the dispersion coefficient is length scale dependent, anomalous dispersion will occur, which is closely analogous to the previously discussed phenomenon of anomalous diffusion in self-similar systems (Gefen et al 1982). We can give a heuristic treatment of how this occurs by first writing how $U$ and $D_{\|}$vary in a random medium as the percolation threshold is approached. For a unit potential gradient (as opposed to the unit potential drop considered above), the flow velocity $U$, in (28), scales as $L^{2} /\langle T\rangle$, where $\langle T\rangle$ is the average transit time for a unit potential drop, and this latter quantity scales as $N_{\mathrm{BB}} / G$. Writing the critical behaviour of these quantities in terms of the length scale, we find that as $L \rightarrow \infty$

$$
\begin{aligned}
& U \sim L^{-\left(t-\beta_{\mathrm{BB}}\right) / \nu} \equiv L^{-\psi} \\
& D_{\|} \sim U L \sim L^{1-\psi} .
\end{aligned}
$$

Now applying scaling arguments, similar to those given by Gefen et al (1982), to describe the critical behaviour of the displacement and spread of a localised pulse of 
tracer, we have

$$
\begin{aligned}
& \frac{\mathrm{d}(x)}{\mathrm{d} t} \sim U \sim L^{-\psi} \\
& \frac{\mathrm{d}}{\mathrm{d} t}\left(\Delta x^{2}\right) \sim D_{\|} \sim L^{1-\psi}
\end{aligned}
$$

where $\langle x\rangle$ is the average position of the tracer, and $\left(\Delta x^{2}\right)=\left\langle x^{2}\right\rangle-\langle x\rangle^{2}$ measures the spatial spread. From our discussion of the homogeneous limit, these results are expected to hold for $x \gg L$. For arbitrary spatial scales, (35) should generalise to the scaling forms

$$
\begin{aligned}
& \frac{\mathrm{d}\langle x\rangle}{\mathrm{d} t} \sim L^{-\psi} \mathrm{g}\left(\frac{\langle x\rangle}{L}\right) \\
& \frac{\mathrm{d}}{\mathrm{d} t}\left(\Delta x^{2}\right) \sim L^{1-\psi} h\left(\frac{\langle x\rangle}{L}\right)
\end{aligned}
$$

where the scaling functions $g(z), h(z) \rightarrow 1$ for $z \gg 1$, and $g(z) \sim z^{-\psi}$ and $h(z) \sim z^{1-\psi}$ for $z \ll 1$. The latter two behaviours follow because dispersion must be insensitive to the value of $L$ when the tracer has traversed a distance which is much less than $L$. For $x \ll L$, we can then integrate (36) to obtain

$$
\begin{aligned}
& \langle x\rangle \sim t^{1 /(1+\psi)} \\
& \left(\Delta x^{2}\right)^{1 / 2} \sim t^{1 /(1+\psi)} .
\end{aligned}
$$

Thus we have both anomalous displacement and anomalous dispersion.

For the displacement, using the known values of the exponents $t, \beta_{\mathrm{BB}}$, and $\nu$, we find that the tracer always moves downstream more slowly than linearly in $t$. The tracer is also found to spread at the same rate as the tracer displacement, in accord with our calculations of the transit time moments. However, from the numerical values of the exponents, we find that the spread of tracer is faster than the $t^{1 / 2}$ rate of a Gaussian process in two dimensions, while above two dimensions, the rate is slower than that of a Gaussian process.

\section{Summary and discussion}

We have introduced a self-similar hierarchical model to describe dispersion in random media in the convective limit. Our model has an adjustable parameter, $\varepsilon$, which controls the relative transit times for different fluid paths across the system. The symmetric version of the model provides an excellent description of the geometry of the backbone of percolation clusters in two dimensions, and we believe that the asymmetric version of the model also has a use in describing geometrical aspects of percolating backbones.

We have found that the average transit time across the system scales exactly as $N_{\mathrm{BB}} / G$, where $N_{\mathrm{BB}}$ is the number of bonds in the backbone and $G$ is the conductance. In calculating the moments of the transit time distribution, we have found two very different classes of behaviour. In the weakly asymmetric case, $\varepsilon>\frac{1}{3}$, all moments of the transit time distribution are governed by a single timescale. This ultimately leads to a dispersion coefficient, $D_{\|}$, which scales as $U L$, where $U$ is the flow velocity and $L$ is the effective system length. This result is reminiscent of $D_{\|} \sim U l$ for a homogeneous 
system, where $l$ is the average grain or pore size. Thus to obtain the dispersion coefficient in a self-similar system, the naive replacement $l \rightarrow L$ is indicated.

For the strongly asymmetric model, a very different type of behaviour is predicted. Dispersion is now dominated by the small fraction of tracer that enters the slowest bond in the network. This leads to $D_{i \mid}$ varying as $U L$ multiplied by a diverging function of $\varepsilon$ as $\varepsilon \rightarrow 0$. This form of the dispersion coefficient is somewhat suggestive of the logarithmic dependence of $D_{\|}$found by Saffman $(1959,1960)$ for dispersion in a homogeneous network model. In such a model, there is a singular correction term modifying the $U L$ dependence which arises from the contribution of the slowest bonds of the network. However, in the Saffman model there is a continuous distribution of very slow bonds, rather than a well defined slowest bond as in the hierarchical model, and integrating over this distribution of slow bonds ultimately leads to a logarithmic correction to $D_{\|}$. In the hierarchical model, however, the well defined value of the slowest traversal time of the network leads to a non-linear power law dependence of $D_{\|}$on $L$. While a definite value of the slowest bond traversal time is an artificial feature of the model, our results do suggest the interesting possibility that the dispersion coefficient does not merely follow from a simple replacement of a microscopic length by a correlation length. Dispersion turns out to be crucially sensitive to the low end of the current distribution in the network, and any correct description of dispersion phenomena on networks with percolation-type disorder will need to account for these details correctly.

\section{Acknowledgments}

As this manuscript was in preparation, we were informed by A Aharony of work by Kutasov et al (1986) in which results similar to those given here were derived in a very different context for a hierarchical Ising system. We are also grateful to E Guyon for a number of very helpful discussions, and to D Stauffer for a critical reading of the manuscript.

\section{References}

Bear J 1971 Dynamics of Fluids in Porous Media (Amsterdam: Elsevier)

Coniglio A 1981 Phys. Rev. Lett. 46250

- 1982 J. Phys. A: Math. Gen. 153829

de Arcangelis L, Koplik J, Redner S and Wilkinson D 1986 Phys. Rev. Lett. 57996

de Arcangelis L, Redner S and Coniglio A 1985 Phys. Rev. B 314725

de Gennes 1983 J. Fluid Mech. 136189

Fried J J and Combarnous 1971 Adv. Hydrosci. 7169

Gefen Y, Alexander S and Aharony A 1982 Phys. Rev. Lett. 5077

Kutasov D, Aharony A, Domany E and Kinzel W 1986 Preprint

Leitzelement M, Maj P, Dodds J A and Greffe J L 1984 Solid -Liquid Separation ed J Gregory (New York: Ellis Horwood)

Mandelbrot B B and Given J 1984 Phys. Rev. Lett. 521853

Michel G 1986 J. Phys. A: Math. Gen. 192461

Roux S, Mitescu C, Charlaix E and Baudet C 1986 J. Phys. A: Math. Gen. 19 L687

Saffman P G 1959 J. Fluid Mech. 6321

1960 J. Fluid Mech. 7194

Sahimi M, Davis H T and Scriven L E 1983 Chem. Eng. Commun. 23329

Sahimi M, Hughes B D, Scriven L E and Davis H T 1986 Chem. Eng. Sci. 412103

Scheidegger A E 1974 The Physics of Flow in Porous Media (Toronto: University of Toronto Press) 